\title{
Taking detection to the limit with optical microcavities: Recent advances presented at the 560. WE Heraeus Seminar
}

\author{
Frank Vollmer ${ }^{1, a}$ and Harald G.L. Schwefel ${ }^{1,2, b}$ \\ 1 Max-Planck-Institute for the Science of Light, Günther-Scharowsky-Str. 1/Bldg. 24, \\ 91058 Erlangen, Germany \\ 2 Institute for Optics, Information and Photonics, University of Erlangen-Nuremberg, \\ Günther-Scharowsky-Str. 1/Bldg. 24, 91058 Erlangen, Germany
}

Received 28 August 2014 / Received in final form 28 August 2014

Published online 6 October 2014

\begin{abstract}
We provide a review on the applications of whispering gallery mode resonators in sensing, and biosensing in particular. We highlight the most recent developments in this area, which were presented at the 560. WE Heraeus Seminar "Taking Detection to the Limit - Biosensing with Optical Microcavities".
\end{abstract}

\section{Introduction}

Optical microcavities are ideal transducers for detecting biomolecules and their interactions: Light confined in a dielectric microstructure, for example by total internal reflection (TIR) in a glass microsphere, is brought to repeated interactions with the biomolecules, thereby enhancing the signal for detection. The internally reflected light traces out its circular optical path many times, exciting a so called whispering gallery mode (WGM). A WGM is an optical resonance localized closely to the rim of the resonator with a high quality $(Q)$ factor, i.e. narrow linewidth [1]. TIR also sets up an evanescent field at the microsphere's surface where the light interacts with the biomolecules in the surrounding medium. Binding of molecules at the microsphere surface causes the WGM resonance frequency to shift. The high $Q$ factor results in a narrow linewidth which allows the detection of minute frequency shifts due to binding of nanoparticles or biomolecules [2-6], Fig. 1. By functionalizing the microsphere surface with specific biomolecular receptors attached to gold nanorods, a WGM transducer can resolve the specific detection of even single oligonucleotides [7], enabling biosensing experiments at the single molecule level.

Biosensors are devices that transduce the interaction events between biomolecules into detectable optical or electrical signals. The analysis of biomolecular interactions is necessary since specific detection in biology relies on monitoring receptor molecules

\footnotetext{
a e-mail: frank.vollmer@mpl.mpg.de

b e-mail: harald.schwefel@mpl.mpg.de
} 

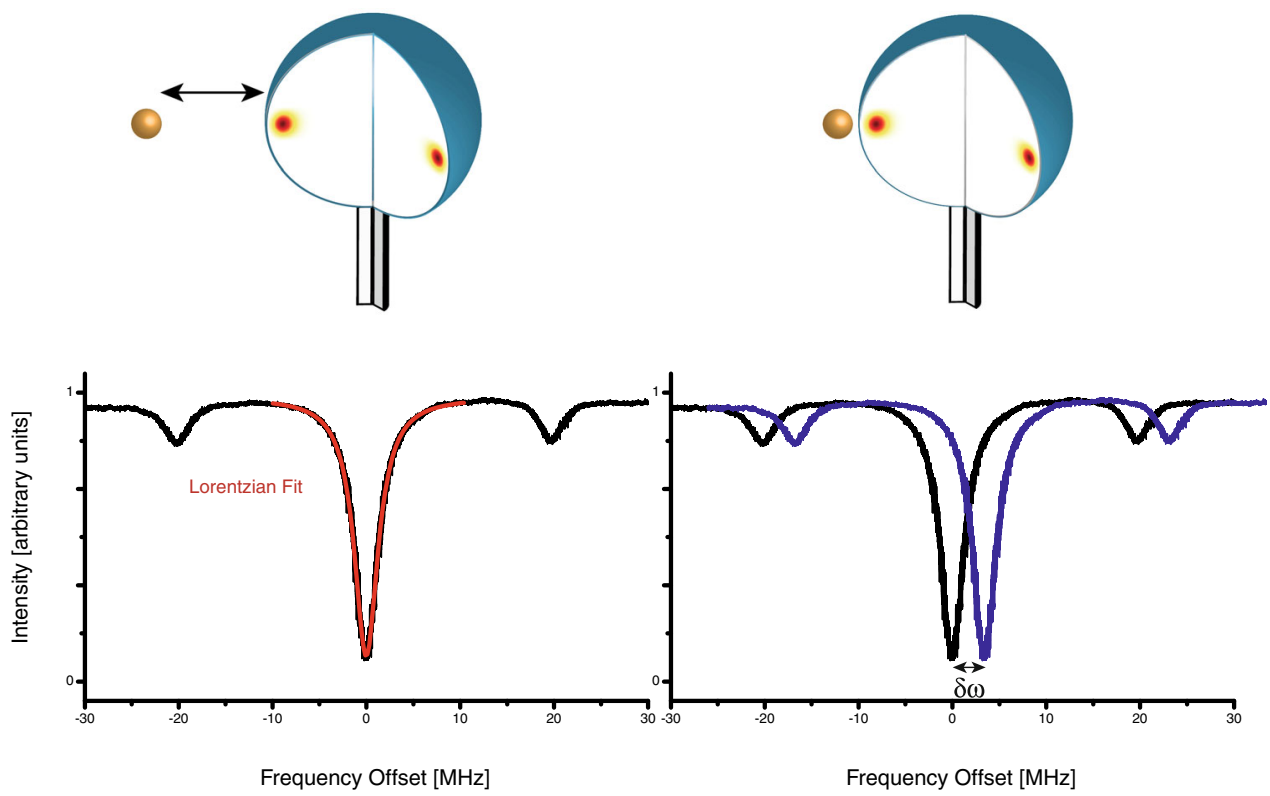

Fig. 1. Left: a high $Q$ whispering gallery mode resonator (here a glass microsphere on a glass fiber stem) exhibits resonances with narrow linewidth. The WGM light field (in yellow/red) is localized close to the rim of the microresonator. Right: upon binding of a molecule or particle a small frequency shift $\delta \omega$ can be measured.

interacting with their target analytes. For example, oligonucleotide receptors are used for specific detection of nucleic acids, and antibodies are used for specific detection of proteins. WGMs enable real time detection of such interactions, with high sensitivity and time resolution [7-13]. However, the applications of WGMs as transducers are by far not only limited to biosensing but they can find broad applications as nanoparticle and virus analyzers [14-17], for measurements of the viscosity of a fluid [18], as refractive index sensors $[19,20]$, monitors of unspecific protein adsorption, down to single proteins $[21,22]$, as metal ion detectors [23], as thermometers [24], detectors of heavy water [25], for determining absolute absorption cross sections [26], tuning of WGMs on-chip $[27,28]$, and for monitoring water adsorption $[29,30]$, to name a few. Developing these varied applications provides a solid ground for improving this technology and cross-fertilizing its different implementations, which taken together have great importance in health care, environmental monitoring, and fundamental studies in the life sciences.

The WGM resonance signal is typically acquired by swept-wavelength scanning of a tunable laser source: a computer controlled external cavity diode laser is scanned, for example every millisecond, to acquire the spectral response of the sphere and determine its resonance frequency, linewidth and amplitude by computer based fitting algorithms [31]. Evanescent field couplers are used to direct the light into the microsphere and excite WGM, examples are prism couplers $[7,32,33]$ and taper and waveguide couplers [3,34-38]. More recently also free space coupled microcavities have been explored in biosensing, which require no coupling device and open up interesting nanoparticle sensing applications by monitoring linewidth change [16]. Also lower $Q$ fluorescent cavities have been explored for WGM biosensing, in which case a spectrometers is used to resolve the fluorescent WGM spectra, with demonstrations of interesting in vitro as well as in vivo applications [39-42]. 


\section{Biodetection mechanisms}

Molecules are most sensitively detected by tracking the shift of WGM frequencies [7]. Theories have been developed to quantitate the frequency shift signals, by tracking one or more WGMs during molecular interaction events, thereby determining the number of molecules bound to the microcavity, deduce their binding orientation, as well as determine the thickness of an adsorbed layer [2,31,34,43-48]. These analysis have been extended to other resonator geometries such as liquid core ring resonators [47], and also many simulation and theoretical tools have been developed in this context [41,49-51]. Quite often, the analysis of relative frequency shift signals in for example ring resonators suffices for many biosensing applications [12,13,17,52]. Another method particularly suitable for nanoparticle analysis, virus detection, and adlayer detection is based on tracking linewidth changes of WGM resonances, where it has been shown that depending on the size of the nanoparticle, the linewidth shift can be more sensitive as compared to the frequency shift [16,26,31]. Furthermore, when operating with particularly high $Q$ resonators, the interaction of WGMs with nanoparticles can result in mode splitting $[14,53]$, and by combining the mode splitting with linewidth measurements it is possible to determine the size of a nanoparticle, independent from its binding location on the microcavity $[14,54,55]$. The mode split analysis is particularly important because it allows the suppression of the common mode noise inherent to both split modes, thereby suppressing for example temperature and bulk refractive index fluctuations. WGMs cannot only be examined in the frequency domain, quite recently approaches in the time domain have been introduced. For example, locking to a WGM mode allows real time detection at high time resolution [56,57]. Furthermore, cavity ring down spectroscopy has been explored with microsphere cavities, opening up the possibility to apply methods established with other cavity geometries to the WGM domain [26,58-60]. Toroidal but also microspherical cavities allow the simultaneous excitation of optical and coupled mechanical modes such that frequency side-bands are created due to the mechanical vibrations, typically in the $\mathrm{MHz}$ to $\mathrm{GHz}$ range $[61,62]$. It has been shown that the mechanical vibrations can be sustained in a liquid core optical ring resonator, a liquid filled capillary that enables the determination of the mechanical properties of a sample, such as viscosity, which can change for example by the amount of dissolved glucose [18]. First steps towards adding Raman spectroscopy to microcavity biosensing were taken recently: trapped particles on a ring resonator were utilized to enhance and collect the Raman signal of a model analyte [63]. Furthermore, the trapping of nanoparticles can find utility in size spectroscopy of particles, and enhancing measurement signals [64-67].

\section{Microresonator geometries, materials and coatings}

Light can be confined resonantly in all kinds of materials and geometries, see Fig. 2. These vast implementation opportunities raise many interesting questions related to the engineering aspects of practical sensor devices. Many different cavity geometries have been explored in this vein, microspheres [68], toroidal shaped cavities [25,69], goblets [70-72], hollow core resonators [47], microbubbles [73,74], bottle resonators [75-80], polymer ring resonators [81-83], silicon ring resonators $[5,84,85]$, silicon ring resonators in reaction tubes and on fiber ends [86,87], as well as other ones.

Material systems range from different glass microcavities, to silicon and silicon nitrate [4]. The ultimate $Q$ factor is material limited and Table 1 gives an overview of $Q$ factors that have been demonstrated for different geometries and materials. The highest achieved $Q$ factors are found within crystalline materials [88]. Crystalline materials are very promising as they can be ultra high $Q$, birefringent, and possess 


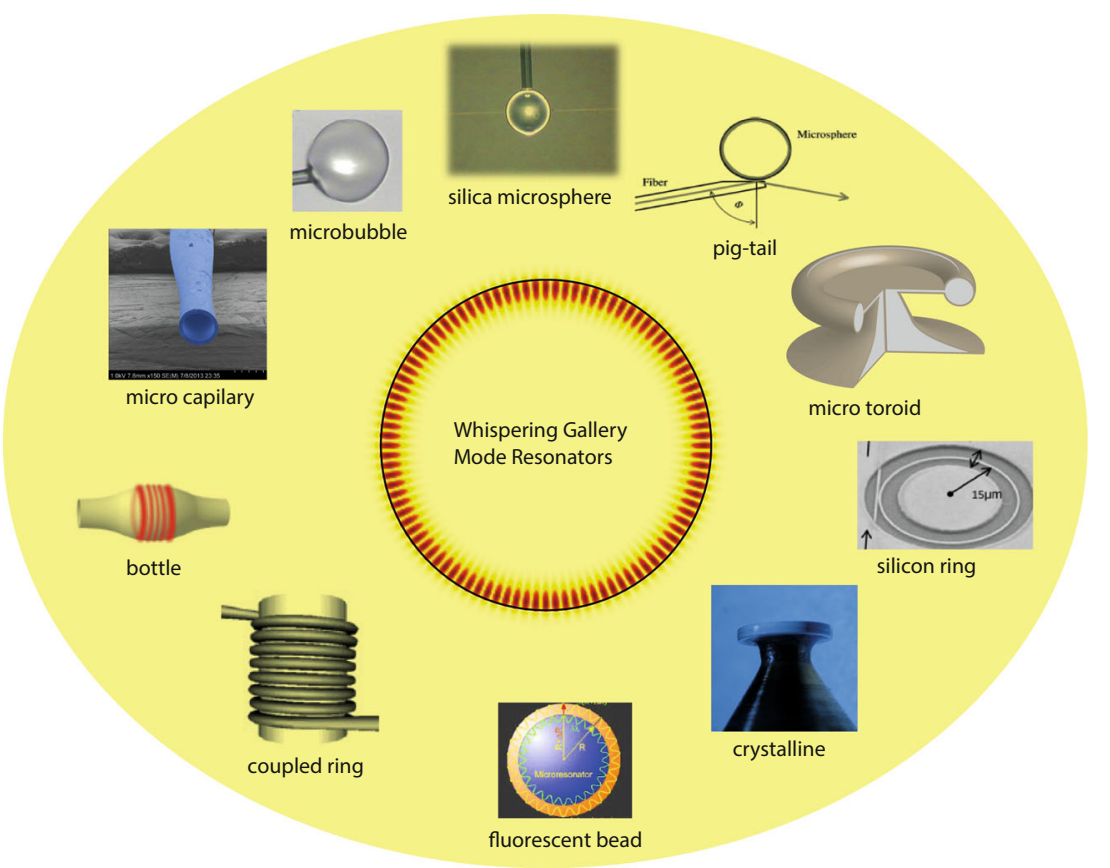

Fig. 2. WGM resonances can be excited in different geometries and material systems.

Table 1. Microcavity $Q$ factor and materials.

\begin{tabular}{llrccl}
\hline \hline Material & $\mathrm{n}$ & Wavelength & $Q_{\text {mat }}$ & $Q_{\exp }$ & Geometry \\
\hline Suprasil & 1.447 & $1310 \mathrm{~nm}$ & $2.3 \times 10^{11}$ & & \\
Suprasil & 1.456 & $656 \mathrm{~nm}$ & $3.0 \times 10^{10}$ & $\sim 9 \times 10^{9}$ & sphere \\
Suprasil & 1.467 & $435 \mathrm{~nm}$ & $9.2 \times 10^{9}$ & & \\
SF-6 & 1.792 & $700 \mathrm{~nm}$ & $7.0 \times 10^{7}$ & $>10^{6}$ & sphere \\
SF-6 & 1.864 & $405 \mathrm{~nm}$ & $1.3 \times 10^{7}$ & & \\
SMF-28 & 1.468 & $1310 \mathrm{~nm}$ & $1.5 \times 10^{11}$ & $>10^{8}$ & sphere \\
SMF-28 & 1.46 & $780 \mathrm{~nm}$ & $2.1 \times 10^{10}$ & $\sim 10^{8}$ & sphere \\
Silicon & 3.476 & $1550 \mathrm{~nm}$ & $1.6 \times 10^{6}$ & $\sim 10^{5}$ & sphere \\
Silicon & 3.476 & $1550 \mathrm{~nm}$ & $1.6 \times 10^{6}$ & $\sim 10^{5}$ & ring \\
CaF $_{2}$ & 1.431 & $795 \mathrm{~nm}$ & $1.1 \times 10^{12}$ & $\sim 10^{11}$ & disk \\
MgF $_{2}$ (ordinary) & 1.375 & $795 \mathrm{~nm}$ & $2 \times 10^{10}$ & $\sim 10^{9}$ & disk \\
MgF $_{2}$ (extraordinary) & 1.387 & $795 \mathrm{~nm}$ & $2 \times 10^{10}$ & $\sim 10^{9}$ & disk \\
Polystyrene & 1.582 & $700 \mathrm{~nm}$ & $4.3 \times 10^{5}$ & $\sim 10^{5}$ & sphere \\
Polystyrene & 1.617 & $435 \mathrm{~nm}$ & $2.6 \times 10^{5}$ & & \\
Polymethylmethacrylate & 1.486 & $700 \mathrm{~nm}$ & $6.4 \times 10^{6}$ & & \\
Polymethylmethacrylate & 1.503 & $435 \mathrm{~nm}$ & $1.1 \times 10^{7}$ & & \\
\hline \hline
\end{tabular}

second order non-linearities. The high quality allows for narrow linewidth lasing [89-91] and the birefringence allows for example for temperature stabilization techniques [92] and complex polarization behaviors [93].

The detection of biomolecules, such as adsorption of bovine serum albumin often serves as a first experiment to test the detection limits of a particular optical microcavity [34,94,95]. For biosensing, the microcavities are further modified with molecular receptors so that the microcavity transduces specific molecular interaction events into an optical signal. Such biosensing demonstrations have been performed 
A)
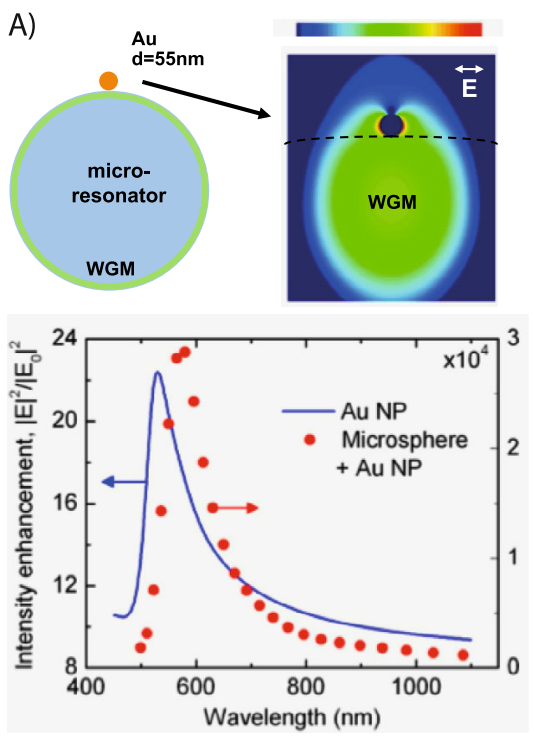

B)
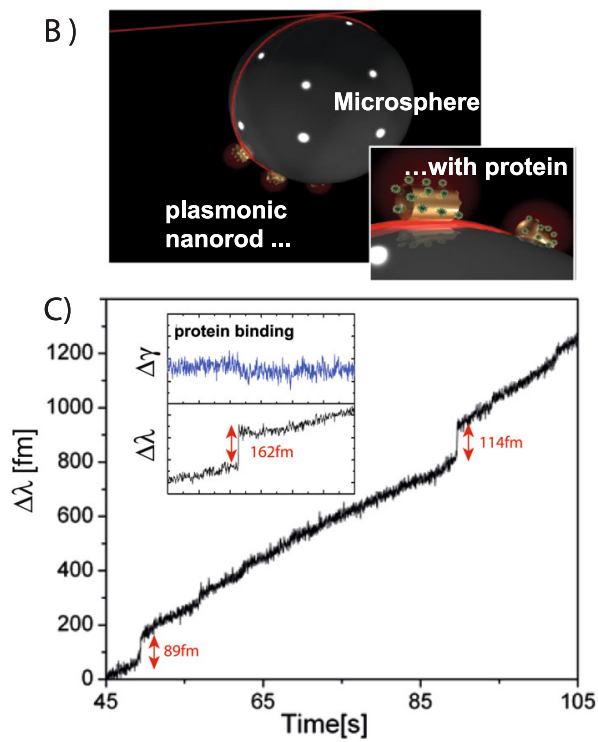

Fig. 3. Plasmonic nanoantennas coupled to microcavities enhance sensitivity in biodetection. A) A molecule binding to a plasmonic nanoantenna coupled to a WGM microcavity experiences field strengths that are enhanced in proportion to $E^{2} / E_{0}^{2}$, boosting the frequency shift upon binding. Adapted from [94]. B) Unspecific adsorption of single BSA proteins to a plasmonic nanoparticle C) appear as steps in the WGM wavelength shift signal $\Delta \lambda$. Inset: as expected, no such steps are resolved in the linewidth trace $\Delta \gamma$, data is taken from [103], for further references see text.

with various receptor molecules including antibodies [8], oligonucleotides [10,12], aptamers [96], immobilized phage proteins [97], etc. In the quest of improving the detection limits towards resolving single molecules, very recently plasmonic enhancements, Fig. 3, have been introduced as means for boosting the frequency shifts in WGM biosensing [49,94,95,98-101], bringing single molecule detection within reach. First demonstrations of enhanced signals have been shown for the unspecific adsorption experiments with bovine serum albumin protein BSA, achieving detection limits down to single proteins and femotomlar concentration levels [22,94,95, 102,103]. Biosensing on the single molecule level was demonstrated by monitoring interactions with specific nucleic acid receptor oligomers, discriminating matching and mismatched strands from their markedly different interaction kinetics [7]. This plasmon enhanced biosensing approach introduces an important paradigm for analysis of single molecules with optical microcavities, requiring the functionalization of a single molecule biosensor with a receptor molecule that interacts with its target molecule only transiently. This allows the proof of single molecule biosensing in experiments that monitor many single molecule interactions for statistical analysis. A Poisson statistics is expected for the time intervals between interaction events, and on-rate constants should follow a linear concentration dependence, governed by a first order rate equation in the single molecule regime [7].

\section{EPJ topical issue "Taking Detection to the Limit"}

The articles in this EPJ special topics issue highlight some of the most recent advances in WGM detection, presented at the 560. WE Heraeus Seminar "Taking Detection to the Limit" - Biosensing with optical microcavities which took place in Bad Honnef, 
Germany in April 2014. The articles are grouped in five sections: I. Hollow Core Resonators. II. WGM Theory. III. WGM Resonator materials. IV. Resonant Geometries/Structures. V. WGM Combined with Fluorescence.

I. Hollow Core Resonators: This minireview by Ward et al. [104] gives an excellent overview of the many applications of hollow whispering gallery microcavity resonator sensors. A tutorial introduction explains the confinement of light in the hollow core, capillary WGM-based resonators, and highlights the sensing mechanisms utilized for these devices. Different fabrication methods and many examples for applications of hollow WGM resonators are presented: pressure sensing, gas and temperature sensing, as well as refractive index- and biosensing. Sensing with liquid core lasers is also reviewed, a very recent approach amenable for WGM biosensing. The minireview is followed by an article by Zhu et al. [105] which shows the utility of hollow WGM resonators as optomechanofluidic sensors. Optical modes interact via mechanical modes excited in the hollow core resonators with a viscous fluid flowing through the core. Numerical simulations are used to estimate the mass sensing capabilities, as well as possibility of extracting other mechanical fluid properties such as density and speed of sound in only nanoliters of sample volume. Sensitivity limits to single particles or cells are given, showing possibility for extracting mechanical properties in biosensing applications using hybrid-shell modes operating in the $\mathrm{MHz}$ to $\mathrm{GHz}$ range.

II. WGM Theory: Yu et al. [106] present a new approach towards numerical analysis of WGM modes which considers the WGMs as straight waveguides, bent to form a closed loop. Analysis of the cavity mode and calculation of $Q$ factor is presented. This method can apply to any WGM structure, and may include metal coatings. The WGM structure in coated microspheres is examined by Ristic et al. [107]. In particular, high refractive index coatings are examined and it is found that the sensitivity to bulk refractive index sensing can be increased by several orders of magnitude. A first experimental realization of a silica microsphere coated with amorphous silicon is shown to have a $Q$ factor of $\sim 4 \times 10^{5}$.

III. WGM Resonator materials: The paper by Foreman et al. [108] introduces liquid droplet WGM resonators for nanoparticle analysis. The concentration of a nanoparticle suspension in the droplets can be detected with higher sensitivity as compared to a glass microsphere immersed in the same nanoparticle solution. Detailed theory is presented, considering sensing mechanisms by frequency and linewidth shifts as well as mode splittings. First experiments show utility of this approach for determining the concentration of gold nanoparticles in a droplet of paraffin oil. Zeltner et al. [109] introduce crystalline disk WGM resonators for refractive index sensing. A $2.9 \mathrm{~mm}$ radius $\mathrm{MgF}_{2}$ disk with a refractive index only slightly higher than water shows refractive index sensitivity of more than $1 \mathrm{~nm} / \mathrm{RIU}$, due to the evanescent field length which is much longer as compared to similar sized silica spheres. Murib et al. [110] introduce a sapphire microsphere for biosensing. WGM spectra are determined from scattered light of a $500 \mu \mathrm{m}$ radius microsphere with $Q \sim 10^{4}$ and an autocorrelation analysis is performed. Application for DNA sensing is discussed. In a similar scattering setup Gökay et al. [111] examine the WGMs of a silicon microsphere resonators and report $Q$ factors up to $10^{5}$ in a $500 \mu \mathrm{m}$ silicon microsphere. Utility for biosensing is discussed.

IV. Resonant Geometries/Structures: The properties of two vertically stacked and coupled silicon ring resonators are discussed by Campanella et al. [112]. The authors use full vectorial 3D FDTD methods in their simulations. The sensitivity to a streptavidin adlayer is predicted. Amini et al. [113] mount fluorescent WGM polystyrene microbeads modified with quantum dots on AFM cantilevers. Their system is used to sense integrin accumulation and formation of focal adhesions at the 
surface of cells. Kosma et al. [42] integrate a $\sim 6 \mu \mathrm{m}$ high index $\mathrm{BaTiO}_{3}$ microsphere inside a microstructured optical fiber which they use to excite WGMs. The microbeads are excited by a supercontinuum laser source and WGMs are detected in scattering spectra acquired using a spectrometer. A $532 \mathrm{~nm}$ "pump" laser is used to demonstrate photorefractive tuning of the modes.

V. WGM Combined with Fluorescence: Bischler et al. [114] present a comprehensive paper on the development of an in vitro diagnostic WGM system based on fluorescent low $Q$ microbeads. WGM analysis, numerical evaluation, sensor conditioning, fluid handling, and control of the overall device are addressed. Peformance of the sensor system is evaluated and compared with other label free techniques such as SPR and QCM. Lastly, Aas et al. [115] report on FRET lasing from self assembled DNA structures excited in droplet resonators. Tetrahedral DNA complexes labeled with $\mathrm{Cy} 3$ and $\mathrm{Cy} 5$ dyes are excited in microdropelts on superhydrophobic surfaces. This work features the combination of DNA nanotechnology with microdroplet lasers to demonstrate an approach for biosensing and novel photonic devices.

FV and HGLS would like to thank the Springer Editorial office, especially Sabine Lehr and Isabelle Houlbert, for their support in editing this special topics issue. They would further like to thank the Max Planck Society and the WE Heraeus Foundation for financial support.

\section{References}

1. K.J. Vahala, Nature 424(6950), 839 (2003)

2. M. Baaske, F. Vollmer, Chem. Phys. Chem. 13(2), 427 (2012)

3. X.D. Fan, I.M. White, S.I. Shopova, H.Y. Zhu, J.D. Suter, Y.Z. Sun, Anal. Chim. Acta 620(1-2), 8 (2008)

4. F. Vollmer, L. Yang, Nanophotonics 1(1), 267 (2012)

5. M. Iqbal, M.A. Gleeson, B. Spaugh, F. Tybor, W.G. Gunn, M. Hochberg, T. BaehrJones, R.C. Bailey, L.C. Gunn, IEEE J. Select. Topics Quant. Electr. 16(3), 654 (2010)

6. H.K. Hunt, A.M. Armani, Nanoscale 2(9), 1544 (2010)

7. M.D. Baaske, M.R. Foreman, F. Vollmer, Nat. Nanotechnol. (2014), doi: $10.1038 /$ nnano.2014.180

8. A.L. Washburn, L.C. Gunn, R.C. Bailey, Anal. Chem. 81(22), 9499 (2009)

9. J.T. Gohring, P.S. Dale, X.D. Fan, Sensors, Actuat. B-Chem. 146(1), 226 (2010)

10. J.D. Suter, D.J. Howard, H.D. Shi, C.W. Caldwell, X.D. Fan, Biosensors Bioelectr. 26(3), 1016 (2010)

11. J.D. Suter, I.M. White, H.Y. Zhu, H.D. Shi, C.W. Caldwell, X.D. Fan, Biosensors Bioelectr. 23(7), 1003 (2008)

12. Y. Wu, D.Y. Zhang, P. Yin, F. Vollmer, Small 10, 2067 (2014)

13. M.S. Luchansky, R.C. Bailey, J. Amer. Chem. Soc. 133(50), 20500 (2011)

14. J.G. Zhu, S.K. Özdemir, Y.F. Xiao, L. Li, L.N. He, D.R. Chen, L. Yang, Nat. Photon. 4(1), 46 (2010)

15. F. Vollmer, S. Arnold, D. Keng, PNAS 105(52), 20701 (2008)

16. L. Shao, X.F. Jiang, X.C. Yu, B.B. Li, W. Clements, F. Vollmer, W. Wang, Y.F. Xiao, Q. Gong, Adv. Mater. 25(39), 5616 (2013)

17. T. Lu, H. Lee, T. Chen, S. Herchak, J.H. Kim, S.E. Fraser, R.C. Flagan, K. Vahala, PNAS 108(15), 5976 (2011)

18. G. Bahl, K.H. Kim, W. Lee, J. Liu, X.D. Fan, T. Carmon, Nat. Comm. 4 (2013)

19. M. Sumetsky, R.S. Windeler, Y. Dulashko, X. Fan, Opt. Express 15(22), 14376 (2007)

20. N.M. Hanumegowda, C.J. Stica, B.C. Patel, I. White, X.D. Fan, Appl. Phys. Lett. $\mathbf{8 7}(20)(2005)$

21. K.A. Wilson, C.A. Finch, P. Anderson, F. Vollmer, J.J. Hickman, Biomaterials 33(1), $225(2012)$ 
22. V.R. Dantham, S. Holler, C. Barbre, D. Keng, V. Kolchenko, S. Arnold, Nano Lett. 13(7), 3347 (2013)

23. S. Panich, K.A. Wilson, P. Nuttall, C.K. Wood, T. Albrecht, J.B. Edel, Anal. Chem. 86(13), 6299 (2014)

24. W.L. Weng, J.D. Anstie, T.M. Stace, G. Campbell, F.N. Baynes, A.N. Luiten, Phys. Rev. Lett. 112(16) (2014)

25. A.M. Armani, K.J. Vahala, Opt. Lett. 31(12), 1896 (2006)

26. J.A. Barnes, G. Gagliardi, H.P. Loock, Optica 1(2), 75 (2014)

27. R. Henze, C. Pyrlik, A. Thies, J.M. Ward, A. Wicht, O. Benson, Appl. Phys. Lett. 102(4), (2013)

28. R. Henze, J.M. Ward, O. Benson, Opt. Express 21(1), 675 (2013)

29. D. Ganta, E.B. Dale, A.T. Rosenberger, Meas. Sci. Technol. 25(5) (2014)

30. L.M. Folan, Appl. Opt. 31(12), 2066 (1992)

31. M.R. Foreman, W. Jin, F. Vollmer, Opt. Express 22(5), 5491 (2014)

32. J. Lutti, W. Langbein, P. Borri, Appl. Phys. Lett. 93(15) (2008)

33. M.L. Gorodetsky, A.A. Savchenkov, V.S. Ilchenko, Opt. Lett. 21(7), 453 (1996)

34. F. Vollmer, D. Braun, A. Libchaber, M. Khoshsima, I. Teraoka, S. Arnold, Appl. Phys. Lett. 80(21), 4057 (2002)

35. M.L. Gorodetsky, V.S. Ilchenko, J. Opt. Soc. A 16(1), 147 (1999)

36. J.C. Knight, G. Cheung, F. Jacques, T.A. Birks, Opt. Lett. 22(15), 1129 (1997)

37. A.J. Qavi, T.M. Mysz, R.C. Bailey, Analytical Chemistry 83(17), 6827 (2011)

38. R. Ismaeel, T. Lee, M. Ding, M. Belal, G. Brambilla, Laser Photon. Rev. 7(3), 350 (2013)

39. M. Himmelhaus, A. Francois, Biosensors Bioelectr. 25(2), 418 (2009)

40. A. Weller, F.C. Liu, R. Dahint, M. Himmelhaus, Appl. Phys. B 90(3-4), 561 (2008)

41. I. Breunig, B. Sturman, F. Sedlmeir, H.G.L. Schwefel, K. Buse, Opt. Express 21(25), $30683(2013)$

42. K. Kosma, I. Konidakis, S. Pissadakis, Eur. Phys. J. Special Topics 223(10), 2035 (2014)

43. S. Arnold, M. Khoshsima, I. Teraoka, S. Holler, F. Vollmer, Opt. Lett. 28(4), 272 (2003)

44. I. Teraoka, S. Arnold, J. Opt. Soc. A 23(7), 1381 (2006)

45. I. Teraoka, S. Arnold, F. Vollmer, J. Opt. Soc. B 20(9), 1937 (2003)

46. M. Noto, F. Vollmer, D. Keng, I. Teraoka, S. Arnold, Opt. Lett. 30(5), 510 (2005)

47. H. Zhu, I.M. White, J.D. Suter, P.S. Dale, X. Fan, Opt. Express 15(15), 9139 (2007)

48. J. Topolancik, F. Vollmer, Biophys. J. 92(6), 2223 (2007)

49. A. Kaplan, M. Tomes, T. Carmon, M. Kozlov, O. Cohen, G. Bartal, H.G.L. Schwefel, Opt. Exp. 21(12), 14169 (2013)

50. F. Vanier, C. La Mela, A. Hayat, Y.A. Peter, Stokes Parameters Measurements for Whispering Gallery Modes Microcavities Characterization (Springer, The Netherlands, 2013), p. 453

51. S. Amini, Y. You, G.W. Kattawar, K.E. Meissner, Appl. Opt. 52(4), 690 (2013)

52. C.E. Soteropulos, H.K. Hunt, A.M. Armani, Appl. Phys. Lett. 99(10) (2011)

53. L. He, S.K. Özdemir, J. Zhu, W. Kim, L. Yang, Nat. Nano 6(7), 428 (2011)

54. L.N. He, S.K. Özdemir, J.G. Zhu, L. Yang, Phys. Rev. A 82(5) (2010)

55. J.G. Zhu, S.K. Özdemir, L. He, D.R. Chen, L. Yang, Opt. Express 19(17), 16195 (2011)

56. J.D. Swaim, J. Knittel, W.P. Bowen, Appl. Phys. Lett. 102(18), 4 (2013)

57. S. Rosenblum, Y. Lovsky, L. Arazi, F. Vollmer, B. Dayan, Nat. Photon. (submitted) (2014)

58. J.A. Barnes, G. Gagliardi, H.P. Loock, Phys. Rev. A 87(5), 053843 (2013)

59. G. Gagliardi, H.P. Loock, Cavity-Enhanced Spectroscopy and Sensing (Springer, Berlin, Heidelberg, 2014)

60. M.I. Cheema, S. Mehrabani, A.A. Hayat, Y.A. Peter, A.M. Armani, A.G. Kirk, Opt. Express 20(8), 9090 (2012) 
61. K. Hyun Kim, G. Bahl, W. Lee, J. Liu, M. Tomes, X. Fan, T. Carmon, Light Sci. Appl. 2, e110 (2013)

62. A. Schliesser, T.J. Kippenberg, Cavity Optomechanics with Whispering-Gallery-Mode Microresonators (Springer, Berlin, Heidelberg, 2014), book section 6, Quantum Science and Technology, p. 121

63. S. Lin, W. Zhu, Y. Jin, K.B. Crozier, Nano Lett. 13(2), 559 (2013)

64. S. Lin, K.B. Crozier, ACS Nano 7(2), 1725 (2013)

65. S. Lin, E. Schonbrun, K. Crozier, Nano Lett. 10(7), 2408 (2010)

66. S. Arnold, D. Keng, S.I. Shopova, S. Holler, W. Zurawsky, F. Vollmer, Opt. Express $\mathbf{1 7}(8), 6230(2009)$

67. Y.F. Chen, X. Serey, R. Sarkar, P. Chen, D. Erickson, Nano Lett. 12(3), 1633 (2012)

68. F. Vollmer, S. Arnold, Nat. Meth. 5(7), 591 (2008)

69. D.K. Armani, T.J. Kippenberg, S.M. Spillane, K.J. Vahala, Nature 421(6926), 925 (2003)

70. U. Bog, T. Laue, T. Grossmann, T. Beck, T. Wienhold, B. Richter, M. Hirtz, H. Fuchs, H. Kalt, T. Mappes, Lab Chip 13(14), 2701 (2013)

71. U. Bog, F. Brinkmann, H. Kalt, C. Koos, T. Mappes, M. Hirtz, H. Fuchs, S. Köber, Small (2014)

72. T. Beck, M. Mai, T. Grossmann, T. Wienhold, M. Hauser, T. Mappes, H. Kalt, Appl. Phys. Lett. 102(12), 121108 (2013)

73. S. Berneschi, A. Barucci, M. Brenci, F. Cosi, D. Farnesi, G.N. Conti, S. Pelli, S. Soria, G. Righini, Optical Microbubble Resonator: A Novel Structure for Sensing Applications (Springer, 2014), p. 359

74. Y. Yang, J. Ward, S.N. Chormaic, Opt. Express 22(6), 6881 (2014)

75. X. Zhang, L. Liu, L. Xu, Appl. Phys. Lett. 104(3), 033703 (2014)

76. A. Rottler, M. Harland, M. Bröll, M. Klingbeil, J. Ehlermann, S. Mendach, Phys. Rev. Lett. 111(25) (2013)

77. M. Sumetsky, Opt. Express 20(20), 22537 (2012)

78. M. Ding, G. Senthil Murugan, G. Brambilla, M.N. Zervas, Appl. Phys. Lett. 100(8), $081108(2012)$

79. G. Senthil Murugan, M.N. Petrovich, Y. Jung, J.S. Wilkinson, M.N. Zervas, Opt. Express 19(21), 20773 (2011)

80. M. Pöllinger, D O'Shea, F. Warken, A. Rauschenbeutel, Phys. Rev. Lett. 103(5) (2009)

81. C. Delezoide, C. Nogues, R. Castro, J. Lautru, M. Buckle, I. Ledoux-Rak, J. Zyss, C.T. Nguyen, in Lasers and Electro-Optics Europe (CLEO EUROPE/IQEC), 2013 Conference on and International Quantum Electronics Conference (IEEE, 2013), p. 1

82. A. Delplanque, E. Henry, J. Lautru, H. Leh, M. Buckle, C. Nogues, Appl. Surf. Sci. (2014)

83. C. Delezoide, M. Salsac, J. Lautru, H. Leh, C. Nogues, J. Zyss, M. Buckle, I. LedouxRak, C.T. Nguyen, IEEE Photon. Techn. Lett. 24(4), 270 (2012)

84. C.A. Barrios, Anal. Bioanal. Chem. 403(6), 1467 (2012)

85. K. De Vos, I. Bartolozzi, E. Schacht, P. Bienstman, R. Baets, Opt. Express 15(12), $7610(2007)$

86. C.L. Arce, S. Van Put, A. Goes, E. Hallynck, P. Dubruel, K. Komorowska, P. Bienstman, J. Appl. Phys. 115(4), 044702 (2014)

87. C.L. Arce, K. De Vos, T. Claes, K. Komorowska, P. Bienstman, SOI Microring Resonator Sensor Integrated on a Fiber Facet (Springer, 2015), p. 53

88. I.S. Grudinin, V.S. Ilchenko, L. Maleki, Phys. Rev. A 74(6), 063806 (2006)

89. B. Sprenger, H.G.L. Schwefel, Z.H. Lu, S. Svitlov, L.J. Wang, Opt. Lett. 35(17), 2870 (2010)

90. J. Alnis, A. Schliesser, C.Y. Wang, J. Hofer, T.J. Kippenberg, T.W. Hänsch, Phys. Rev. A 84(1), 011804 (2011)

91. M.C. Collodo, F. Sedlmeir, B. Sprenger, S. Svitlov, L.J. Wang, H.G.L. Schwefel, Opt. Express 22(16), 19277 (2014) 
92. D. Strekalov, R. Thompson, L. Baumgartel, I. Grudinin, N. Yu, Opt. Express 19(15), 14495 (2011)

93. F. Sedlmeir, M. Hauer, J.U. Fürst, G. Leuchs, H.G.L. Schwefel, Opt. Express 21(20), $23942(2013)$

94. M.A. Santiago-Cordoba, S.V. Boriskina, F. Vollmer, M.C. Demirel, Appl. Phys. Lett. 99(7) (2011)

95. M.A. Santiago-Cordoba, M. Cetinkaya, S.V. Boriskina, F. Vollmer, M.C. Demirel, J. Biophoton. 5(8-9), 629 (2012)

96. H.Y. Zhu, J.D. Suter, I.M. White, X.D. Fan, Sensors 6(8), 785 (2006)

97. H. Chibli, H. Ghali, S. Park, Y.A. Peter, J.L. Nadeau, Analyst 139(1), 179 (2014)

98. J.D. Swaim, J. Knittel, W.P. Bowen, Appl. Phys. Lett. 99(24) (2011)

99. Y.F. Xiao, Y.C. Liu, B.B. Li, Y.L. Chen, Y. Li, Q.H. Gong, Phys. Rev. A 85(3) (2012)

100. B.K. Min, E. Ostby, V. Sorger, E. Ulin-Avila, L. Yang, X. Zhang, K. Vahala, Nature 457(7228), 455 (2009)

101. S.I. Shopova, R. Rajmangal, S. Holler, S. Arnold, Appl. Phys. Lett. 98(24) (2011)

102. V.R. Dantham, S. Holler, V. Kolchenko, Z. Wan, S. Arnold, Appl. Phys. Lett. 101(4) (2012)

103. F. Vollmer, (2014) http://www.mpg.de/research/yearbooks/2014?search=vollmer

104. J.M. Ward, N. Dhasmana, S.N. Chormaic, Eur. Phys. J. Special Topics 223(10), 1917 (2014)

105. K. Zhu, K. Han, T. Carmon, X. Fan, G. Bahl, Eur. Phys. J. Special Topics 223(10), $1937(2014)$

106. W. Yu, S. Herchak, T. Lu, Eur. Phys. J. Special Topics 223(10), 1949 (2014)

107. D. Ristić, A. Chiappini, M. Mazzola, D. Farnesi, G. Nunzi-Conti, G.C. Righini, P. Féron, G. Cibiel, M. Ferrari, M. Ivanda, Eur. Phys. J. Special Topics 223(10), 1959 (2014)

108. M.R. Foreman, S. Avino, R. Zullo, H.-P. Loock, F. Vollmer, G. Gagliardi, Eur. Phys. J. Special Topics 223(10), 1971 (2014)

109. R. Zeltner, F. Sedlmeir, G. Leuchs, H.G.L. Schwefel, Eur. Phys. J. Special Topics 223(10), 1989 (2014)

110. M. Murib, Y. Yílmaz, A. Demir, Ş. Işçi, T. Bilici, A. Kurt, P. Wagner, A. Serpengüzel, Eur. Phys. J. Special Topics 223(10), 1995 (2014)

111. U.S. Gökay, M. Zakwan, A. Serpengüzel, Eur. Phys. J. Special Topics 223(10), 2003 (2014)

112. C.E. Campanella, C.M. Campanella, F. De Leonardis, V.M.N. Passaro, Eur. Phys. J. Special Topics 223(10), 2009 (2014)

113. S. Amini, Z. Sun, G.A. Meininger, K.E. Meissner, Eur. Phys. J. Special Topics 223(10), 2023 (2014)

114. R. Bischler, M. Olszyna, M. Himmelhaus, L. Dähne, Eur. Phys. J. Special Topics 223(10), 2041 (2014)

115. M. Aas, E. Özelci, A. Jonáš, A. Kiraz, H. Liu, C. Fan, Q. Chen, X. Fan, Eur. Phys. J. Special Topics 223(10), 2057 (2014) 